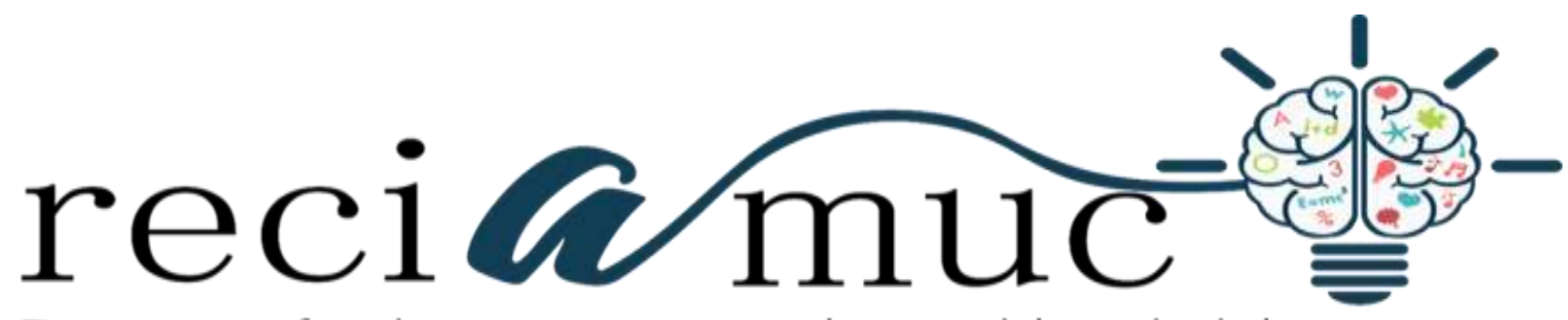

Revista cientifica de investigación actualización del mundo de las ciencias

Jheison David Cabrera Ruilova ${ }^{\text {a; }}$ Melissa Paulina Pereira Ponton ${ }^{\text {b; }}$ Rossibell

Berenisse Ollague Armijos ${ }^{c}$; María Monserrate Ponce Ventura ${ }^{\text {d }}$

Factores de riesgo de preeclampsia

Risk factors for preeclampsia

Revista Científica de Investigación actualización del mundo de las Ciencias. Vol. 3

núm., 2, abril, ISSN: 2588-0748, 2018, pp. 1012-1032

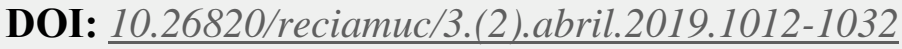

URL: http://reciamuc.com/index.php/RECIAMUC/article/view/384

Código UNESCO: 3205 Medicina Interna

Tipo de Investigación: Artículo de Revisión

(c) RECIAMUC; Editorial Saberes del Conocimiento, 2019

Recibido: $15 / 01 / 2019$

Aceptado: 10/03/2019

Publicado: 01/04/2019

Correspondencia: jheisoncabrera.r@gmail.com

a. Médico; Investigador Independiente; Guayaquil, Ecuador; jheisoncabrera.r@gmail.com

b. Médico; Investigador Independiente; Guayaquil, Ecuador; melissapereirap@ outlook.es

c. Médico; Investigador Independiente; Guayaquil, Ecuador; dra_ollague@ hotmail.com

d. Licenciada en Terapia Física, Médico, Tecnóloga Médica en Terapia Física y Rehabilitación; Investigador Independiente; Guayaquil, Ecuador; ruthgilerzambrano@gmail.com 


\section{Factores de riesgo de preeclampsia}

Vol. 3, núm. 2., (2019)

Jheison David Cabrera Ruilova; Melissa Paulina Pereira Ponton; Rossibell Berenisse Ollague

Armijos; María Monserrate Ponce Ventura

\section{RESUMEN}

La preeclampsia es la presión arterial alta y signos de daño hepático o renal que ocurren en las mujeres después de la semana 20 de embarazo. A través de una serie de investigaciones de tipo documental que lleva como objetivo el profundizar acerca del riesgo que corren las mujeres que padecen de este tipo de hipertensión arterial (HTA) en el embarazo. Destacando que las mujeres que padecen de preeclampsia durante el embarazo, acarrean toda una serie de complicaciones más, tales como la obesidad, o algún daño a cualquier otro órgano. $O$ incluso el simple hecho de tener antecedentes de preeclampsia en la familia trae consecuencias negativas tanto para la madre como para él bebe. Cabe mencionar que tales daños ocasionados a los órganos son, malformaciones cardiacas, malformaciones de los pulmones, problemas de sangrado, convulsiones (eclampsia), falla hepática, ACV, o en los casos más graves incluso la muerte para ambos. Tomando en cuenta factores como la condición física de la madre antes del embarazo, como, por ejemplo, el peso, la raza, la edad o incluso antecedentes de preeclampsia en la familia, y otros factores más que pueden contribuir con el desarrollo de la misma. Acotando así que, Si bien es poco frecuente, la preeclampsia también se puede presentar en una mujer después de dar a luz a su bebé, casi siempre dentro de las siguientes 48 horas. Esto se denomina preeclampsia posparto. Lo cual es importante tener en cuentan si se ha padecido se esta complicación en el embarazo.

Palabras claves: Preeclampsia; Hipertensión arterial; Eclampsia; HELLP; Diabetes gestacional. 


\title{
Factores de riesgo de preeclampsia
}

Vol. 3, núm. 2., (2019)

Jheison David Cabrera Ruilova; Melissa Paulina Pereira Ponton; Rossibell Berenisse Ollague Armijos; María Monserrate Ponce Ventura

\begin{abstract}
Preeclampsia is high blood pressure and signs of liver or kidney damage that occur in women after the 20th week of pregnancy. Through a series of documentary-type research that aims to deepen the risk of women suffering from this type of arterial hypertension (AHT) in pregnancy. Stressing that women who suffer from preeclampsia during pregnancy, carry a whole series of complications, such as obesity, or some damage to any other organ. Or even the simple fact of having a history of preclamation in the family has negative consequences for both the mother and the baby. It is worth mentioning that such damages caused to the organs are, cardiac malformations, malformations of the lungs, bleeding problems, seizures (eclampsia), liver failure, stroke, or in the most severe cases even death for both. Taking into account factors such as the physical condition of the mother before pregnancy, such as, for example, weight, race, age or even a history of preeclampsia in the family, and other factors that may contribute to the development of the same. Limiting so, while it is rare, preeclampsia can also occur in a woman after giving birth to her baby, almost always within the next 48 hours. This is called postpartum preeclampsia. Which is important to consider if you have suffered this complication in pregnancy.
\end{abstract}

Key words: Preeclampsia; Arterial hypertension; Eclampsia; HELLP; Gestational diabetes. 


\section{Factores de riesgo de preeclampsia}

Vol. 3, núm. 2., (2019)

Jheison David Cabrera Ruilova; Melissa Paulina Pereira Ponton; Rossibell Berenisse Ollague Armijos; María Monserrate Ponce Ventura

\section{Introducción.}

Las diferentes investigaciones coinciden que esta complicación que se presenta en el embarazo tiene una la probabilidad de desarrollarse en mujeres que tienes antecedentes cardiovasculares ya bien sea en ellas mismas o en la familia, aparte de la obesidad y factores como la edad de menos de 20 a mayores de 35 años, aparte de que uno de los mayores riesgos que se pueden presentar es que la sintomatología se pueda presentar a la mitad o al inicio del embarazo.

La preeclampsia es un trastorno hipertensivo considerablemente común durante el embarazo, de presentación progresiva, su causa aún es desconocida y acarrea con frecuencia graves complicaciones maternas y perinatales. (Huamán, 2016)

La incidencia de esta patología es amplia ya que influyen numerosos aspectos como la localización geográfica, la raza, los factores nutricionales o inmunológicos, las comorbilidades, y hasta el clima húmedo y frío se ha relacionado con una mayor incidencia de mujeres afectadas. Se estima que alrededor del 7\% de los embarazos desarrollarán una preeclampsia, aunque ésta puede ser mayor énfasis en entornos socioeconómicos más desfavorecidos y en países donde existe una prevalencia de enfermedades cardiovasculares. Si nos referimos a poblaciones de mujeres blancas en países desarrollados, esta cifra es notablemente más exagerada. Estudios hospitalarios en el norte de Europa de mujeres seguidas desde el inicio de la gestación sugieren que la incidencia de preeclampsia se sitúa alrededor del $2 \%$ en primerizas. (Torres, Vega, Vinalay, Cortaza, \& Gutiérrez, 2018) 


\section{Factores de riesgo de preeclampsia}

Vol. 3, núm. 2., (2019)

Jheison David Cabrera Ruilova; Melissa Paulina Pereira Ponton; Rossibell Berenisse Ollague Armijos; María Monserrate Ponce Ventura

El 5\% de las preeclampsias evolucionarán, finalmente, como una eclampsia, y hasta en un 19\% pueden hacerlo como un síndrome HELLP (hemolysis, elevated liver enzymes, and low platelets), lo que se asocia con más complicaciones y mayor mortalidad. Los resultados maternales adversos en la preeclampsia se deben, fundamentalmente, a una disfunción en el sistema nervioso central, hepático o renal (accidente cerebrovascular hemorrágico, ruptura hepática o fracaso renal agudo) y al sangrado asociado a trombocitopenia. Una de las 3 causas más frecuentes de muertes en embarazadas son la preeclampsia-eclampsia, además de las enfermedades tromboembólicas y el sangrado. En Estados Unidos, la tasa de mortalidad materna debida a la preeclampsia-eclampsia es aproximadamente de 1 por cada 100.000 nacimientos vivos, lo que origina una mortalidad fetal de 6,4 casos por cada 10.000 partos. Otros factores que influyen negativamente en el pronóstico de estas pacientes son la edad gestacional, la severidad de la aparición y factores coexistentes con la gestación como la diabetes, gestación múltiple, enfermedad renal previa al embarazo, trombofilia o hipertensión preexistente. El pronóstico neonatal deriva de una mayor prematuridad en los casos en los que se indica finalización anticipada de la gestación, crecimiento intrauterino retardado o abrupción de placenta. En la preeclampsia leve-moderada, el pronóstico suele ser bueno, comparable a gestaciones con complicaciones consideradas normales, salvo por la mayor tasa de inducción o intervención durante el parto, mientras que en las preeclampsias severas sí se ha observado una mayor tasa de disfunción hepática y renal en la madre, además de nacimiento prematuro, crecimiento retardado y dificultad respiratoria en los neonatos debido a malformaciones pulmonares, aunque esto no se asoció a mayor mortalidad fetal o neonatal. El mayor riesgo de morbilidad, tanto para la madre como para el feto, se ha visto en las preeclampsiaseclampsias que aparecen en el segundo trimestre de gestación, probablemente debido a una mayor 


\section{Factores de riesgo de preeclampsia}

Vol. 3, núm. 2., (2019)

Jheison David Cabrera Ruilova; Melissa Paulina Pereira Ponton; Rossibell Berenisse Ollague Armijos; María Monserrate Ponce Ventura

influencia de prematuridad y bajo peso en el caso del feto, y un tratamiento más conservador en el caso de la madre para mayor maduración. (Curiel, Prieto, \& Mora, 2008)

\section{Materiales y Métodos}

Las herramientas y materiales de trabajo que se conciben para el desarrollo de este trabajo investigativo son los siguientes: computadores personales con conexión a internet y un cúmulo de contenidos científico-académicos diversos, con los que se persigue aportar información de calidad y actualizado respecto los Factores de Riesgo de la Preeclampsia. Se escoge un diseño bibliográfico, en el marco de una metodología de revisión sistemática.

Esta investigación se enfoca en la búsqueda y revisión sistemática de literatura científicoacadémica seleccionada, que por una parte, está disponible determinadas bases de datos, entre las que figuran: MedlinePlus, PubMed, Biblioteca Virtual de la Salud (BVS), SciELO, Dialnet y ELSEVIER, Cochrane, entre otras; y por la otra, mediante el uso del material físicamente disponible en la biblioteca universitaria como en otras particulares, representando todo ello parte esencial del proceso investigativo y de comprensión que facilita la síntesis de la mejor evidencia disponible, y a su vez representando todo ello uno de los resultados esperados.

Principalmente se realiza una búsqueda aleatoria y consecutiva en las mencionadas bases de datos, usando las expresiones "preeclampsia", "presión arterial alta", "factores de Riesco de la preeclampsia" y "eclampsia”, lo que aproximadamente resultó en más de un centenar de miles de registros bibliográficos. Luego éstos se filtran en base a criterios de: idioma español, relevancia, correlación temática y fecha de publicación en los últimos siete años, sin descartar por tipo de 


\section{Factores de riesgo de preeclampsia}

Vol. 3, núm. 2., (2019)

Jheison David Cabrera Ruilova; Melissa Paulina Pereira Ponton; Rossibell Berenisse Ollague Armijos; María Monserrate Ponce Ventura

material bibliográfico; es decir, se escogen títulos de artículos científicos, ensayos, revisiones sistemáticas, protocolos, editoriales, libros, boletines, folletos, tesis de grado, posgrado y doctorado, noticias científicas, entre otros documentos e información de interés científico y académico.

Seguidamente, se efectúa la selección y clasificación de la literatura científica académica físicamente disponible, aplicando, en términos generales, los criterios antes mencionados, siendo a partir de allí que el equipo investigador le da la correspondiente lectura crítica y análisis de toda esa evidencia científica, lo que resultó consecutiva y consensuadamente en el fundamento de las ideas y planteamientos aquí plasmados.

\section{Resultados}

La preeclampsia generalmente comienza después de las 32 semanas de embarazo está caracterizada por presión arterial alta y presentando signos de daños en otro sistema de órganos, más frecuentemente el hígado y los riñones en mujeres cuya presión arterial había sido normal anteriormente. Si no se trata a tiempo, la preeclampsia puede acarrear una serie de graves complicaciones, incluso mortales, tanto para la madre como para el bebé. Si se tiene preeclampsia, el tratamiento más efectivo es dar a luz al bebé. Si se diagnostica preeclampsia en el embarazo demasiado temprano para dar a luz a tu bebé, la madre y el médico enfrentan a una tarea difícil. El bebé necesita más tiempo para madurar, pero se debe evitar poner a la o al bebé en riesgo de sufrir complicaciones graves. En ocasiones muy poco frecuentes, la preeclampsia se desarrolla después del parto, una enfermedad que se conoce como preeclampsia posparto. (Vallejo, Landázuri, Loor, \& Vallejo, 2019) 


\section{Factores de riesgo de preeclampsia}

Vol. 3, núm. 2., (2019)

Jheison David Cabrera Ruilova; Melissa Paulina Pereira Ponton; Rossibell Berenisse Ollague Armijos; María Monserrate Ponce Ventura

\section{¿Cuántas mujeres tienen o corren riesgo de tener preeclampsia?}

De acuerdo a el Instituto Nacional de la Salud Infantil y Desarrollo Humano Eunice Kennedy Shriver de Estados Unidos de América [NICHD, por sus siglas en inglés] (s.f.), se desconoce la cantidad exacta de mujeres que desarrollan preeclampsia. Algunos científicos y profesionales de la salud estiman que la preeclampsia afecta a entre el 5\% y el 10\% de todos los embarazos a nivel mundial. Las tasas son más bajas en los Estados Unidos (aproximadamente el $3 \%$ al $5 \%$ de las mujeres), pero se estima que representa entre el $40 \%$ y el $60 \%$ de las muertes maternas en los países en desarrollo. En estos países, los trastornos relacionados con la presión arterial alta son la segunda causa de nacimiento de niños muertos y muerte neonatal temprana.

Además, el síndrome HELLP se presenta en entre el 10\% y el 20\% de todas las mujeres con preeclampsia grave o eclampsia. (NICHD, s.f.)

Según la Organización Mundial de la Salud, en las mujeres que tuvieron preeclampsia, entre el $20 \%$ y el $40 \%$ de sus hijas y entre el $11 \%$ y el $37 \%$ de sus hermanas también tendrá el trastorno.

La preeclampsia es más común en las mujeres con antecedentes de determinados problemas de salud, como las migrañas, la artritis reumatoide, el lupus, la esclerodermia, las infecciones del tracto urinario, las enfermedades de las encías, el síndrome del ovario poliquístico, la esclerosis múltiple, la diabetes gestacional y la anemia drepanocítica. La preeclampsia también es más común en los embarazos que son el resultado de la donación de óvulos, la inseminación con semen de donante o la fecundación in vitro. (NICHD, s.f.) 


\section{Factores de riesgo de preeclampsia}

Vol. 3, núm. 2., (2019)

Jheison David Cabrera Ruilova; Melissa Paulina Pereira Ponton; Rossibell Berenisse Ollague Armijos; María Monserrate Ponce Ventura

\section{Síntomas}

A veces, la preeclampsia es asintomática. La presión arterial alta puede presentarse lentamente o tener una aparición repentina. En los cuidados prenatales una parte imprescindible es el control de la presión arterial porque el primer signo de preeclampsia es, con frecuencia, el aumento de la presión arterial. La presión arterial que supera los 140/90 milímetros de mercurio, resulta ser anormal.

Otros signos y síntomas de preeclampsia pueden comprender:

- Exceso de proteínas en la orina (proteinuria) u otros signos de problemas renales

- Dolores de cabeza intensos

- Cambios en la visión, entre ellos, pérdida temporal de la visión, visión borrosa o sensibilidad a la luz, similares a los síntomas de la migraña.

- Dolor en la parte superior del abdomen, por lo general, debajo de las costillas y en el lado derecho

- Náuseas o vómitos

- Menor producción de orina

- Niveles más bajos de plaquetas en la sangre (trombocitopenia)

- Función hepática alterada

- Dificultad para respirar, causada por la presencia de líquido en los pulmones 


\section{Factores de riesgo de preeclampsia}

Vol. 3, núm. 2., (2019)

Jheison David Cabrera Ruilova; Melissa Paulina Pereira Ponton; Rossibell Berenisse Ollague Armijos; María Monserrate Ponce Ventura

La preeclampsia puede provocar aumento de peso repentino e hinchazón (edema), producida por la retención de líquidos especialmente en el rostro y en las manos. No obstante, esto también ocurre en muchos embarazos normales, por lo que no se consideran signos fiables de preeclampsia. (Mayo Clinic, 2018)

\section{Causas}

La causa exacta de la preeclampsia comprende varios factores. Se cree que esta complicación comienza en la placenta, el cual es el órgano que nutre al feto durante el embarazo. Durante las primeras semanas del embarazo, se forman nuevos vasos sanguíneos y estos evolucionan para enviar sangre a la placenta de forma eficiente.

En las mujeres con preeclampsia, parece que estos vasos sanguíneos tal vez no se desarrollan o no funcionan de manera adecuada. Son más angostos que los vasos sanguíneos normales y reaccionan de manera diferente a las indicaciones hormonales, lo cual causa que la cantidad de sangre que puede fluya de manera limitada a través de ellos.

Las causas de esta formación anormal pueden comprender las siguientes:

- Un flujo de sangre insuficiente al útero.

- Daño en los vasos sanguíneos.

- Un problema en el sistema inmunitario.

- Determinados genes.

(Mayo Clinic, 2018) 


\section{Factores de riesgo de preeclampsia}

Vol. 3, núm. 2., (2019)

Jheison David Cabrera Ruilova; Melissa Paulina Pereira Ponton; Rossibell Berenisse Ollague Armijos; María Monserrate Ponce Ventura

Factores de riesgo

La Fundación Mayo para la Educación Médica, [MFMER, por sus siglas en ingles] (2018), establece que la preeclampsia se manifiesta solo como una complicación del embarazo. Los factores de riesgo incluyen los siguientes:

- Antecedentes de preeclampsia. Los antecedentes personales o familiares de preeclampsia aumentan significativamente el riesgo de tener preeclampsia.

- Hipertensión crónica. Si se padece de hipertensión crónica antes del embarazo, se corre mayor riesgo de desarrollar preeclampsia.

- Primer embarazo. El riesgo de padecer preeclampsia es mayor durante el primer embarazo.

- Nueva paternidad. Cada embarazo con una pareja nueva aumenta más el riesgo de tener preeclampsia que tener un segundo o un tercer embarazo con la misma pareja.

- La edad. El riesgo de tener preeclampsia es mayor en el caso de las mujeres embarazadas muy jóvenes o mayores de 40 años.

- La raza. Las mujeres de raza negra tienen un riesgo más alto de tener preeclampsia que las de otras razas.

- Obesidad. El riesgo de preeclampsia es mayor si la gestante es obesa.

- Embarazo múltiple. La preeclampsia es más frecuente en las mujeres embarazadas de mellizos, trillizos u otros embarazos múltiples.

- Intervalo entre embarazos. Tener bebés con menos de dos años o más de 10 años de diferencia conduce a un mayor riesgo de tener preeclampsia. 


\section{Factores de riesgo de preeclampsia}

Vol. 3, núm. 2., (2019)

Jheison David Cabrera Ruilova; Melissa Paulina Pereira Ponton; Rossibell Berenisse Ollague Armijos; María Monserrate Ponce Ventura

- Antecedentes de determinadas afecciones. Tener determinadas afecciones antes de quedar embarazada, como presión arterial alta crónica, migrañas, diabetes tipo 1 o tipo 2, enfermedad renal, una tendencia a tener coágulos sanguíneos o lupus, aumenta el riesgo de tener preeclampsia.

- Fecundación in vitro. El riesgo de tener preeclampsia aumenta si la concepción del bebé fue mediante fecundación in vitro.

Otros factores de riesgo de la preeclampsia son los que se relacionados con: La genética, por haber padecido preeclampsia en algún embarazo previo; El sistema inmunitario, debido al poco tiempo de exposición al semen de la pareja; la madre; el tabaquismo, estrés o trabajos pesados, padecimiento de enfermedades renales, inmunológicas, o alteraciones de la coagulación; el propio embarazo, mola vesicular, anomalías fetales congénitas, padecimiento de infección urinaria durante el embarazo.

Por supuesto, todos estos factores no son determinantes, es decir, ninguno por sí solo o combinados pueden causar preeclampsia de forma infalible, sino que simplemente están relacionados con su aparición aumentando las posibilidades de padecerla. (MFMER, 2018)

Los factores de riesgo de preeclampsia han sido clasificados o divididos de diferente manera por varios autores. Así, Cruz, Hernández, Yanes, \& Isla (2019) en base a otros tratadistas, dicen que, están los que las dividen en: genéticos y medioambientales; los que las clasifican en preconcepcionales o crónicos y vinculados con el embarazo. Por lo tanto, refirieron que; 


\section{Factores de riesgo de preeclampsia}

Vol. 3, núm. 2., (2019)

Jheison David Cabrera Ruilova; Melissa Paulina Pereira Ponton; Rossibell Berenisse Ollague Armijos; María Monserrate Ponce Ventura

En otros estudios epidemiológicos se ha llegado a la conclusión que son modificables y no modificables, visión que pudiera ser más operativa y práctica, ya que acepta o da la opción de cambiar algunos de ellos, en este caso, los que se consideran modificables. También se acepta la división en placentarios y maternos. (párr. 3)

Así mimo, aportan una clasificación propia que se divide en maternos, y éstos a su vez en preconcepcionales y relacionados con la gestación en curso; y medioambientales.

\section{Maternos:}

Preconcepcionales y relacionados con la gestación en curso:

- Edad materna menor de 20 y mayor de 35 años.

- Historia personal de PE (en embarazos anteriores).

- Presencia de algunas enfermedades crónicas: hipertensión arterial, obesidad, diabetes mellitus, resistencia a la insulina, enfermedad renal, neurofibromatosis, síndrome anti fosfolípido primario (anticuerpos anti fosfolípidos) y otras enfermedades autoinmunes (síndrome anti fosfolípido secundario), trombofilias y dislipidemia.

Relacionados con la gestación en curso:

- Primigravidez o embarazo de un nuevo compañero sexual.

- Sobredistención uterina (embarazo gemelar y poli hidramnios).

- Embarazo molar en nulípara.

Ambientales: 


\section{Factores de riesgo de preeclampsia}

Vol. 3, núm. 2., (2019)

Jheison David Cabrera Ruilova; Melissa Paulina Pereira Ponton; Rossibell Berenisse Ollague Armijos; María Monserrate Ponce Ventura

- Malnutrición por defecto o por exceso.

- Escasa ingesta de calcio previa y durante la gestación.

- Hipomagnesemia y deficiencias de zinc y selenio.

- Alcoholismo durante el embarazo.

- Bajo nivel socioeconómico.

- Cuidados prenatales deficientes.

- Estrés crónico.

(Cruz, Hernández, Yanes, \& Isla, 2019)

Cuando se produce la fecundación del ovocito (precursor inmaduro del ovulo), por el espermatozoide del hombre se forma un óvulo que lleva consigo la información genética mezclada de ambos padres. Este óvulo muy pronto comienza a dividirse, llega a las paredes del útero, y las invade para poder implantarse en su interior. La invasión depende de la futura placenta, que se llama trofoblasto y que, en condiciones normales, es capaz de integrarse completamente en el espesor de la pared uterina hasta que alcanza los vasos sanguíneos que aportarán sangre a la futura placenta.

Sin embargo, en las mujeres que desarrollan preeclampsia, la invasión no se produce correctamente, y no se alcanzan completamente los vasos sanguíneos; esto sucede porque el sistema inmunitario de la madre rechaza al trofoblasto, cuya genética depende directamente del espermatozoide paterno. Al no conseguir alcanzar correctamente los vasos sanguíneos, se produce una situación de isquemia a lo largo del embarazo; es decir, insuficiencia de sangre para toda la placenta y algunas células mueren liberando toxinas y desechos celulares que pasan a la 


\section{Factores de riesgo de preeclampsia}

Vol. 3, núm. 2., (2019)

Jheison David Cabrera Ruilova; Melissa Paulina Pereira Ponton; Rossibell Berenisse Ollague Armijos; María Monserrate Ponce Ventura

circulación, alterando a arterias y arteriolas. Esto provoca una contracción difusa del aparato circulatorio y aparece la hipertensión arterial. (Corralo, 2019)

\section{Complicaciones}

Mientras más grave sea la preeclampsia y más temprano se presente en el embarazo, mayores serán los riesgos para la madre y el bebé. La preeclampsia puede requerir la inducción del trabajo de parto produciendo un parto prematuro. Puede ser necesaria una cesárea en caso de complicaciones clínicas u obstétricas que requieran un parto rápido. De tal manera, es posible que el médico recomiende un parto vaginal programado. El obstetra tiene el deber ayudar a la madre a decidir qué tipo de parto es adecuado para la enfermedad, puesto depende de en qué momento se presente la complicación de la preeclampsia y que tan avanzada este.

Un estudio realizado a 197 paciente, 101 con preeclampsia (51,3\%) las cuales presentan un proceso de agravamiento de preeclampsia, y a 96 controles sin preeclampsia (48,7\%). Concluyendo el estudio se determinó que las pacientes que sentaban este agravamiento también presentaban un índice de masa corporal mayor a la del paciente que tenían un control prenatal normal, este estudio determinó que el sobre peso y la obesidad contribuyen al desarrollo de la preeclampsia en el embarazo y agravamiento en las mujeres que presentaban HTA antes del embarazo o aquellas que ya tenían antecedentes de preeclampsia. (Vivian \& Martos, 2017)

Existen diversos estudios que indican una relación entre el ácido úrico sérico y el hecho de que contribuye al hecho de que se desarrolle la preeclampsia, ocasionando un bajo peso en él bebe. 


\section{Factores de riesgo de preeclampsia}

Vol. 3, núm. 2., (2019)

Jheison David Cabrera Ruilova; Melissa Paulina Pereira Ponton; Rossibell Berenisse Ollague Armijos; María Monserrate Ponce Ventura

La complicación crea problemas tanto para la madre como para el feto, y afecta a entre $2 \%$ y $7 \%$ de los embarazos.

Theimer (2017) refiere que (Garovic, 2017), respecto a la investigación en la que participó, aseguró que descubrieron:

[...] que la preeclampsia continúa persiguiendo a las madres, mucho tiempo después de haber dado a luz a sus hijos. Lo bueno es que estos resultados pueden servir para intervenir pronto en los factores de riesgo, antes de que se presente una enfermedad cardiovascular. (Theimer, 2017)

El sistema de coagulación está activado, posiblemente debido a la disfunción de las células endoteliales que produce la activación plaquetas. El síndrome HELLP (hemólisis, pruebas de función hepática elevadas y bajo recuento de plaquetas) aparece en el 10 al 20\% de las mujeres con preeclampsia grave o eclampsia; esta incidencia es de 100 veces la de los embarazos normales (1 a 2/1.000). La mayoría de las mujeres embarazadas con síndrome HELLP tienen hipertensión y proteinuria, pero algunas no tienen ninguna de ellas. (Dulay, 2017).

No obstante, (Corominas, Balconi, Palermo, Maskin, \& Damiano, 2014) acota que:

No observamos una relación directa ni con el sexo fetal ni con el tiempo de aparición de los síntomas clínicos. Estos hallazgos sugieren que los cambios en las concentraciones de ácido úrico se deberían a alteraciones en los estadios iniciales de la preeclampsia. Por ello, la monitorización de los niveles del mismo durante el embarazo podría contribuir al abordaje precoz de este desorden gestacional. 


\section{Factores de riesgo de preeclampsia}

Vol. 3, núm. 2., (2019)

Jheison David Cabrera Ruilova; Melissa Paulina Pereira Ponton; Rossibell Berenisse Ollague Armijos; María Monserrate Ponce Ventura

Entre las complicaciones de la preeclampsia se incluyen las siguientes:

$\checkmark$ Restricción del crecimiento fetal. La preeclampsia afecta a las arterias que suministran sangre a la placenta. Si la placenta no recibe la cantidad suficiente de sangre, el bebé puede recibir un nivel inadecuado de sangre y oxígeno, y menos nutrientes. Esto puede ocasionar un crecimiento lento, conocido como "restricción del crecimiento fetal", bajo peso al nacer o parto prematuro.

$\checkmark$ Parto prematuro. Si se tiene preeclampsia con características graves, es posible que se deba adelantar el parto para salvar la vida de la y la del bebé. Si el bebé nace prematuro, puede tener problemas respiratorios o de otro tipo. El médico de cabecera ayudará a la madre a comprender cuál es el momento ideal para el parto.

$\checkmark$ Desprendimiento placentario. La preeclampsia aumenta el riesgo de tener desprendimiento de la placenta, un trastorno que consiste en la separación de la placenta de la pared interior del útero antes del parto. Un desprendimiento grave puede ocasionar un sangrado intenso, que puede poner en riesgo la vida de la madre y la del bebé.

$\checkmark$ Síndrome de HELLP. El síndrome de HELLP, cuyas siglas significan hemólisis (la destrucción de los glóbulos rojos), aumento de enzimas hepáticas y recuento bajo de plaquetas, es una forma más grave de la preeclampsia, y puede poner en riesgo rápidamente tu vida y la del bebé.

$\checkmark$ Los síntomas del síndrome de HELLP incluyen náuseas y vómitos, dolores de cabeza, y dolor en la parte superior derecha del abdomen. El síndrome de HELLP es particularmente peligroso porque implica daños a varios sistemas de órganos. En ocasiones, puede 


\section{Factores de riesgo de preeclampsia}

Vol. 3, núm. 2., (2019)

Jheison David Cabrera Ruilova; Melissa Paulina Pereira Ponton; Rossibell Berenisse Ollague Armijos; María Monserrate Ponce Ventura

manifestarse repentinamente, incluso antes de que se detecte la presión arterial alta, o sin ningún síntoma en absoluto.

$\checkmark$ Eclampsia. Si la preeclampsia no se controla, es posible que se desarrolle una eclampsia, que es, en esencia, una preeclampsia con convulsiones. Es muy difícil predecir qué pacientes tendrán una preeclampsia lo suficientemente grave para convertirse en eclampsia.

$\checkmark$ Con frecuencia no hay síntomas ni signos de advertencia con los que se pueda anticipar la eclampsia. Dado que la eclampsia puede tener consecuencias graves para la madre y el bebé, el parto se vuelve necesario, independientemente del tiempo de gestación del embarazo.

$\checkmark$ Daño a otros órganos. La preeclampsia puede dañar los riñones, el hígado, los pulmones, el corazón o los ojos, y puede causar un accidente cerebrovascular u otra lesión cerebral. La cantidad de lesiones causadas a otros órganos depende de la gravedad de la preeclampsia.

$\checkmark$ Enfermedad cardiovascular. Tener preeclampsia puede aumentar el riesgo de presentar enfermedades cardíacas y de los vasos sanguíneos (cardiovasculares) en el futuro. El riesgo se vuelve incluso mayor si se ha tenido preeclampsia más de una vez o si se tuvo un parto prematuro. Para minimizar el riesgo, después del parto intentar mantener un peso ideal, comer una variedad de frutas y verduras, haz ejercicio de manera regular y no fumes.

Comer menos sal, cambiar tus actividades, restringir calorías o consumir ajo o aceite de pescado no reduce el riesgo. No se ha demostrado que un aumento de la ingesta de vitaminas $\mathrm{C}$ y E sea beneficioso. 


\section{Factores de riesgo de preeclampsia}

Vol. 3, núm. 2., (2019)

Jheison David Cabrera Ruilova; Melissa Paulina Pereira Ponton; Rossibell Berenisse Ollague Armijos; María Monserrate Ponce Ventura

Algunos estudios han revelado una relación entre la deficiencia de vitamina D y un mayor riesgo de tener preeclampsia. Sin embargo, mientras algunos estudios han demostrado una relación entre tomar suplementos de vitamina D y un menor riesgo de tener preeclampsia, otros no han logrado establecer la conexión.

No obstante, en ciertos casos, se puede reducir el riesgo de tener preeclampsia con lo siguiente:

$\checkmark$ Aspirina en dosis bajas. Si se tiene ciertos factores de riesgo, que incluyen antecedentes de preeclampsia, embarazo múltiple, presión arterial alta crónica, enfermedad renal, diabetes o enfermedad autoinmune, el médico puede recomendar una dosis diaria baja de aspirina (81 miligramos) a partir de las 12 semanas de embarazo.

$\checkmark$ Suplementos de calcio. En algunas poblaciones, las mujeres que tienen deficiencia de calcio antes del embarazo, y que no obtienen suficiente calcio durante el embarazo a través de sus dietas, podrían beneficiarse de los suplementos de calcio para prevenir la preeclampsia. Sin embargo, es poco probable que las mujeres de los Estados Unidos u otros países desarrollados tengan una deficiencia de calcio en un grado tal que los suplementos de calcio las beneficien. (Curiel, Prieto, \& Mora, 2008)

\section{Conclusión}

Los antecedentes de estudios de la preeclampsia concluyen que problemas como los cardiometabólicos como la obesidad, hipertensión arterial, (Suárez \& Gutierrez, 2019) concluyen en su investigación contribuyen como un factor de riesgo de la misma, ocasionando así una serie 


\section{Factores de riesgo de preeclampsia}

Vol. 3, núm. 2., (2019)

Jheison David Cabrera Ruilova; Melissa Paulina Pereira Ponton; Rossibell Berenisse Ollague Armijos; María Monserrate Ponce Ventura

de consecuencias tanto para el feto como para la gestante. Tomando en cuenta que los estudios arrojan que un aumento de en la masa corporal o en el ácido úrico sérico durante el embarazo pueden también producir esta condición o empeorarla si ya se tiene un previo diagnóstico de la misma, aparte de la edad de la madre que está estrechamente vinculada al desarrollo de preeclampsia, lo cual hace reflexionar de que se debe tener un control antes, durante y después del embarazo para que se pueda gozar de buena salud sin este tipo de complicaciones.

\section{Referencias bibliográficas}

Corominas, A., Balconi, S., Palermo, M., Maskin, B., \& Damiano, A. (Diciembre de 2014). Portal Regional de la BVS. Recuperado el 23 de 07 de 2019, de Portal Regional de la BVS: http://hdl.handle.net/11336/11627

Corralo, D. S. (05 de Marzo de 2019). webconsultas.com. Obtenido de webconsultas.com: https://www.webconsultas.com/embarazo/complicaciones-del-embarazo/causas-de-lapreeclampsia

Cruz, J., Hernández, P., Yanes, M., \& Isla, A. (13 de agosto de 2019). Factores de riesgo de preeclampsia: enfoque inmunoendocrino. Parte I. Revista Cubana de Medicina General Integral, 23(4). Recuperado el 26 de 07 de 2019, de http://scielo.sld.cu/scielo.php?script=sci_arttext\&pid=S0864-21252007000400012

Curiel, E., Prieto, M., \& Mora, J. (Junio de 2008). Factores relacionados con el desarrollo de preeclampsia. Revisión de la bibliografía. Clínica e Investigación en Ginecología y Obstetricia, 35(3), 87-97. doi:10.1016/S0210-573X(08)73053-3

Dulay, A. T. (Octubre de 2017). msdmanuals.com. Obtenido de msdmanuals.com: https://www.msdmanuals.com/es-ve/professional/ginecolog\%C3\%ADa-yobstetricia/anomal\%C3\%ADas-del-embarazo/preeclampsia-y-eclampsia

Huamán, C. (2016). Prevalencia de factores de riesgo para la preeclampsia en mujeres atendidas en el Hospital Nacional Dos de Mayo entre enero a junio 2015. Lima: Universidad Ricardo Palma. Recuperado el 24 de 07 de 2019, de https://core.ac.uk/download/pdf/58916763.pdf

Mayo Clinic. (16 de Noviembre de 2018). mayoclinic.ORG. Recuperado el 24 de 07 de 2019, de mayoclinic.ORG: https://www.mayoclinic.org/es-es/diseasesconditions/preeclampsia/symptoms-causes/syc-20355745

MFMER. (16 de noviembre de 2018). Mayo Clinic. Recuperado el 27 de 07 de 2019, de Mayo Clinic: https://www.mayoclinic.org/es-es/diseases-conditions/preeclampsia/symptomscauses/syc-20355745

NICHD. (s.f.). nichd.nih.gov. ( Instituto Nacional de la Salud Infantil y Desarrollo Humano Eunice Kennedy Shriver (NICHD)) Recuperado el 25 de 07 de 2019, de nichd.nih.gov: 


\section{Factores de riesgo de preeclampsia}

Vol. 3, núm. 2., (2019)

Jheison David Cabrera Ruilova; Melissa Paulina Pereira Ponton; Rossibell Berenisse Ollague Armijos; María Monserrate Ponce Ventura

https://www1.nichd.nih.gov/espanol/salud/temas/preeclampsia/informacion/Pages/riesgos -madre.aspx

Suárez, J., \& Gutierrez, M. (2019). Caracterización del riesgo cardiometabólico en mujeres de edad mediana con antecedentes de preeclampsia en la última década. CorSalud, 11(1), 30 - 36. Recuperado el 24 de 07 de 2019, de http://www.revcorsalud.sld.cu/index.php/cors/article/view/407

Theimer, S. (29 de agosto de 2017). Las madres con antecedentes de preeclampsia pueden tener problemas cardiovasculares más adelante en la vida. Notiia Electrónica, Mayo Clinic, News Network, Rochester. Minnesota. Recuperado el 23 de 07 de 2019, de newsnetwork.mayoclinic.org: https://newsnetwork.mayoclinic.org/discussion/las-madrescon-antecedentes-de-preeclampsia-pueden-tener-problemas-cardiovasculares-masadelante-en-la-vida/

Theimer, S. (29 de agosto de 2017). newsnetwork.mayoclinic.org. Obtenido de newsnetwork.mayoclinic.org: https://newsnetwork.mayoclinic.org/discussion/las-madrescon-antecedentes-de-preeclampsia-pueden-tener-problemas-cardiovasculares-masadelante-en-la-vida/

Torres, M., Vega, E., Vinalay, I., Cortaza, L., \& Gutiérrez, A. (Julio-Septiembre de 2018). Factores de riesgo psicosociales asociados a preeclampsia en mujeres mexicanas: análisis comparado en tres Estados. Enfermeria Universitaria, 15(3), 226-243. doi:10.22201/eneo.23958421e.2018.3.65987

Vallejo, A., Landázuri, J., Loor, K., \& Vallejo, K. (2019). La preeclampsia - eclampsia. El fantasma latente en las mujeres embarazadas. Recimundo, 3(2). doi:https://doi.org/10.26820/recimundo/3.(2).abril.2019.566-581

Vivian, A., \& Martos, F. (Abril - Junio de 2017). El sobrepeso y la obesidad como factores de riesgo para la preeclampsia. revista cuba ginecologia y obstetricia, 43(2), 1 - 11. $\begin{array}{lllllll}\text { Recuperado } & \text { el } & 25 & \text { de } & 07 & \text { de } & 2019,\end{array}$ http://scielo.sld.cu/scielo.php?script=sci_arttext\&pid=S0138-600X2017000200007

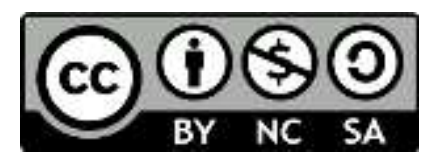

RECONOCIMIENTO-NOCOMERCIAL-COMPARTIRIGUAL

CC BY-NC-SA

ESTA LICENCIA PERMITE A OTROS ENTREMEZCLAR, AJUSTAR Y CONSTRUIR A PARTIR DE SU OBRA CON FINES NO

COMERCIALES, SIEMPRE Y CUANDO LE RECONOZCAN LA AUTORÍA Y SUS NUEVAS CREACIONES ESTÉN BAJO UNA LICENCIA CON LOS MISMOS TÉRMINOS. 